\title{
NUMEN Y DEUS EN LA CONCEPCIÓN RELIGIOSA DE AMIANO MARCELINO
}

\author{
Narciso SANTOS YANGUAS \\ Universidad de Oviedo
}

\begin{abstract}
RESUMEN: Entre los términos utilizados por Amiano para referirse al concepto de divinidad el lugar preeminente lo ocupa numen, al que se añaden otros calificativos (deus...). La actividad desplegada por este poder se manifiesta sensible y eficaz: la divinidad otorga a los hombres los favores de su solicitud, justicia, benevolencia y beneficencia. El historiador parece creer en una omnipresencia del elemento divino en el desarrollo de las acciones de los hombres. Como manifestación de ese principio los sacrificios implican una concepción activa como forma suprema de petición a los dioses y de tenerlos propicios.
\end{abstract}

PALABRAS CLAVE: Numen, deus, Amiano, divinidad.

ABSTRACT: Numen appears to be the principal term among those used by Ammianus when he refers to the concept of divinity. It also appears associated to other terms, like for example deus. The activity of this power is characterized by a high degree of sensitivity and reliability: divinity offers men its favours in the form of justice, benevolence and charity. The historian appears thus to believe in the ever present intervention of divine elements in the actions that men carry out. Sacrifices imply in this sense an active and supreme offering to the gods with the aim of having them on our side.

KEYWORDS: Numen, deus, Ammianus, concept of divinity.

\section{INTRODUCCIÓN}

La realidad histórica que nos describe el historiador de Antioquia se ajusta a un relato sometido a tensión dramática, lo que se vería agravado a su vez por la precipitación de los acontecimientos y se traduciría en una especie de angustia universal $^{1}$.

Como consecuencia de ello el sentido de lo trágico en las Res Gestae no sería más que el resultado del conflicto que se vislumbra y desarrolla a lo largo de ella

\footnotetext{
${ }^{1}$ Ver, por ejemplo, FonTAINE, J. (1969). "Ammien Marcellin, historien romantique". BAGB, p. 417 y ss.
} 
entre Virtus y las fuerzas irracionales, personificadas en la Fortuna y el fatum ${ }^{2}$.

Ahora bien, no vamos a referirnos aquí al análisis de la consideración del cristianismo en el marco del sentido religioso del historiador antioqueno ${ }^{3}$.

Tras un somero repaso a las principales interpretaciones que se han llevado a cabo desde mediados del siglo pasado acerca de la concepción amianea del cristianismo $^{4}$, es posible afirmar que nuestro historiador se manifestaría con discreción y ambigüedad a un mismo tiempo con respecto al tema, calificando al cristianismo como una "religión absoluta y simple" (Amm. Marc. 21.16.18: Christianam religionem absolutam et simplicem $)^{5}$.

En su descripción histórica de los acontecimientos el historiador de Antioquía deja de lado por lo general los conflictos teológicos y religiosos, si hacemos excepción de muy escasos ejemplos, como el enfrentamiento que se produce en Roma entre Dámaso y Ursino por la posesión de la sede episcopal (Amm.Marc. 27.3.12) $)^{6}$.

A este respecto es posible afirmar que el cristianismo, en aquellos momentos muy próximo a convertirse en la religión oficial del Estado romano a manos de Teodosio, aparece sin embargo en las Res Gestae amianeas caracterizado como una realidad casi extraña y perjudicial ${ }^{7}$.

Por ello algunos investigadores han llegado a pensar que, entre el politeísmo tradicional y el monoteísmo cristiano, nuestro historiador ocuparía una posición

2 A. SELEM. "Il senso del tragico in Ammiano", ASNP 34 81965) 404 y ss. Cf. igualmente N. SANTOS, "Fortuna y fatum: la contingencia en el desarrollo de la historia según Amiano Marcelino" (en prensa).

${ }^{3}$ Entre otros motivos porque ya lo hemos llevado a cabo con anterioridad. Cf. N. SANTOS. (1996). "Ammiano Marcelino, Teodosio y el cristianismo". HAnt, 20, p. 437 y ss.

${ }^{4}$ Ver, entre otros, S. D’EliA. (1962). "Ammiano Marcellino ed il cristianesimo". Stud Rom, 10, p. 372 y ss.; A. DEMANDT. (1965). Zeitkritik und Geschichtsbild im Werke Ammia. Bonn, pp.80-85; F. PASCHOUd. (1967). Roma Aeterna. Roma, pp. 47-49, y W. ENSSLIN. (1971). Zur Geschichtsschreibung und Weltanschauung des Ammianus Marcellinus. Aalen,, p. 96.

${ }^{5}$ V. NERI. (1992). "Ammianus". Definition of Christianity as absoluta et simplex religio". En Cognitio gestorum. Amsterdam, pp. 59 y ss.

${ }^{6}$ L. Angliviel De La Baumelle. (1974). "Remarques sur l'attitude d'Ammien Marcellin à l'égard du christianisme". En Mélanges à W. Seston. París, pp. 15 y ss.

7 Más detalles en A. SELEM. (1964). "Considerazioni circa Ammiano Marcellino ed il cristianesimo". RCCM, 6 p. 224 y ss. 
bastante original, que nos resulta relativamente fácil de definir si tomamos como punto de referencia la complejidad del vocabulario utilizado en su obra ${ }^{8}$.

\section{EL CONCEPTO DE LO DIVINO}

Entre los términos utilizados comúnmente por Amiano Marcelino para referirse al concepto de divinidad el lugar preeminente viene ocupado por numen.

Sin embargo, ello no es óbice para que, en los sucesivos libros conservados de su obra, vaya desgranando igualmente los calificativos de supernum (Amm.Marc. 14.11.24. Ver igualmente 15.2.8 у 16.12.18 у 62), summum (Amm.Marc. 15.8.9. Ver también 17.7.3; 21.13.14 y 26.6.9), sempiternum (Amm.Marc. 17.13.28; 25.3.19; 28.5.19 y 31.10.18) ${ }^{10}$, celeste (Amm.Marc. 19.1.4. Ver igualmente 16.12.13; 26.1.5; 27.6.8 y 31.16.4) ${ }^{11}$, superum (Amm.Marc. 25.8.3), divino (Amm.Marc.26.1.14. Ver igualmente 19.10.4), perpetuum (Amm.Marc. 27.3.15. Ver también 29.2.20) y magnum (Amm.Marc.29.5.40).

De cualquier forma la actividad desplegada por este poder de carácter divino se manifestaría en todas las ocasiones de una manera sensible y eficaz (Amm.Marc. 15.11.25).

Como consecuencia de ello la divinidad se nos muestra dispensando a los hombres los favores de su solicitud (secunda o propitia voluntas) (Amm.Marc. 14.10.2 y 27.6.8), así como de su justicia (aequitas) (Amm.Marc. 14.11.24), de su benevolencia (favor) (Amm.Marc. 16.12.62; 21.13.14 y 25.8.3) y de su beneficiencia (benignitas) (Amm.Marc. 21.1.9).

A ello hemos de añadir que, en ciertas ocasiones, se manifestaría igualmente complaciente y haría brotar en el interior de los mortales resoluciones sabias (placida ope numinis) (Amm.Marc.24.4.1).

Veamos la aplicación de estos principios a algunos ejemplos de los que recoge el historiador de Antioquía en su obra: inmediatamente después de la muerte de Galo, Juliano habría perecido bajo los esfuerzos combinados de los cortesanos, confabulados contra su persona, si la emperatriz Eusebia, guiada por una

\footnotetext{
${ }^{8}$ P. M. CAMUS. (1967). Ammien Marcellin, témoin des courants culturels et religieux à la fin du IVe siècle, París, p.134.

9 A. Grenier. (1974). "Numen. Observations sur l'un des éléments primordiaux de la religion romaine", Latomus, p. 297 y ss.

${ }^{10}$ Más detalles en M. L. W. LAISTNER. ( 1971). The Greater Roman Historian. Londres , p.159.

${ }^{11}$ S. JannaCone, S. (1960). Ammiano Marcellino. profilo storico-critico. Nápoles, p.79.
} 
inspiración divina, no hubiera intercedido ante Constancio II en su favor (Amm.Marc. 15.2.8) ${ }^{12}$.

Por ello cuando el propio Constancio presenta a Juliano ante los soldados como César, la asamblea militar proclama que su elección obedecía más a una inspiración divina que a un deseo humano (Amm.Marc. 15.8.9: arbitrium summi numinis id esse $)^{13}$.

Y, finalmente, con términos similares se expresa el historiador antioqueno cuando nos describe la elección de Valentiniano I (Amm.Marc. 26.1.5: numinis adspiratione caelestis electus).

Asimismo sería esta fuerza proveniente del numen la que asistiría a los soldados romanos en sus expediciones guerreras: en 3 ocasiones parece afirmarse, por ejemplo, la ayuda divina en favor de Juliano durante su campaña contra los alamanes en el año 357, con anterioridad a la batalla de Argentoratus y en el transcurso de la misma (Amm.Marc. 16.12.18 y 52 y 62$)^{14}$.

De esta manera la cura caelestis, el favor caelestis, en definitiva el auxilio divino, es decir la intervención de los dioses a favor de los romanos, aparece reseñada en diversas ocasiones (ver, como ejemplo, Amm.Marc. 18.3.1).

En dicho contexto sobresale de forma especial la ayuda prestada en enfrentamientos bélicos determinantes para el futuro de la Ciudad, ya se trate del Genio que empuja al combate y conduce a los soldados de Juliano hacia la victoria en su lucha contra los alamanes (Amm.Marc. 16.12.13), o bien del ser colosal que en el año 363 se lanzó al asalto de la ciudad de Maogamalcha llevando en sus manos una escala y a quien nadie encontró nunca (Amm.Marc.24.4.24), o finalmente de la presencia de una divinidad tutelar que se hizo visiblemente manifiesta junto al emperador ${ }^{15}$.

12 J. BIDEZ. (1965). La vie de l'empereur Julien, París, pp.108-109.

13 R. C. Blockley. (1972). "Constantius Gallus and Jullian as Caesars of Constantius II". Latomus, 31, p. 446.

${ }^{14}$ N. SANTos. (1976). Los pueblos germánicos en la segunda mitad del siglo IV d.C. Oviedo, pp.33 y ss.

${ }^{15}$ Más detalles sobre estos episodios en M. MesLin. (1974). "Le merveilleux comme langage politique chez Ammien Marcellin". En Mélanges à W. Seston. París, p.362. 
Además, sería la propia Roma la que se iba a ver protegida, desde el momento mismo de su nacimiento como Estado, hasta el punto de que se le aseguraría una duración eterna (Amm.Marc. 19.1.4).

No obstante, este poder divino se mostraría en ocasiones como algo hostil y contrario al hombre (laevum numen), destacando por ejemplo el momento en que el César Galo, guiado por una divinidad contraria, abandona la ciudad de Antioquía, siendo asesinado poco después por orden de Constancio (Amm.Marc. 14.11.12) ${ }^{16}$.

Y esta misma oposición de la divinidad a los intereses de los mortales se iba a poner de realce con motivo de la invasión de los godos en el año 376 (Amm.Marc. 31.4.9) ${ }^{17}$.

\section{PAPEL DEL NUMEN EN EL DESARROLLO DE LOS ACONTECI- MIENTOS}

Esta fuerza divina se nos muestra en las Historias de Amiano como un elemento justificativo de los acontecimientos, aunque en ciertas ocasiones la imprecisión a la hora de emplear los términos numen o deus con el fin de referirse al elemento divino sea ostensible y ambos vocablos sirvan indistintamente para hacer alusión a un mismo concepto ${ }^{18}$.

Ahora bien, la utilización de deus como un simple equivalente de numen resulta, sin embargo, muy poco frecuente en el relato amianeo.

Frente a ello, todo un conjunto de ejemplos puede inclinarnos a pensar que, de acuerdo con el cristianismo de los personajes que hacen uso del mismo, se corresponde con el dios cristiano: así lo encontramos utilizado en este sentido al menos en dos ocasiones en boca de Constancio (Amm.Marc. 15.8.10 y 14), e incluso el mismo Juliano, antes de su definitiva conversión al paganismo tradicional, se refiere igualmente al dios del cielo y cuenta para su empresa con la ayuda conjunta de sus soldados y de dicha divinidad (Amm.Marc. 21.5.3 y 5).

Sin duda el caso más llamativo de todos ellos lo constituye el hecho de que Valentiniano I ponga como garantía absoluta de sus éxitos la ayuda de Dios (Amm.Marc. 27.7.6). Pero estos mismos personajes utilizan a veces el término

\footnotetext{
${ }^{16}$ R. MoONEY. (1958). "Gallus Caesar's Last Journey", CPh , 53, pp. 175-177.

${ }^{17}$ N. Bagliv. (1995). Ammianea. Catania, 1995, pp.115 y ss.

${ }^{18}$ P. M. CAMUS. (1967). Ammien Marcellin, témoin des courants culturels et religieux à la fin du IVe siècle, pp.133 y ss.
} 
numen junto al de deus: de este modo lo vamos a encontrar, por ejemplo, al menos en dos ocasiones, con motivo de las arengas de Constancio (Amm.Marc. 14.10.12 y 21.13.14).

En cuanto a Juliano, en el transcurso de su estancia en territorio galo, finge asociarse a las devociones de los cristianos, hasta tal punto que en la Epifanía del año 361 toma parte directamente en las ceremonias religiosas invocando solemnemente a dicha divinidad (Amm.Marc. 21.2.5) ${ }^{19}$.

Finalmente Valentiniano, con motivo de la presentación del nuevo Augusto Graciano a las tropas, hace alusión en su discurso al deus, así como, poco después, a las disposiciones favorables de la divinidad celeste (Amm.Marc. 27.6.6 y 8).

Este mismo emperador, al contraponer el lujo de los obispos de Roma a la simplicidad de vida que llevaban los prelados de las provincias, hace subrayar el hecho de que éstos se encomiendan por sus propios méritos a la benevolencia de la divinidad eterna y de sus verdaderos adoradores (Amm.Marc. 27.3.15).

Incluso en ocasiones ambos términos del vocabulario (deus y numen) aparecen asociados con referencia a una misma expresión, designando a la divinidad suprema sin ninguna otra precisión (Amm.Marc. 25.7.5).

Como conclusión podemos afirmar que no contamos con pruebas suficientes, en el marco de la terminología religiosa utilizada por el historiador antioqueno, para establecer una distinción clara entre ambos vocablos, que sirven para designar de manera indiferente el poder celeste, siendo utilizado numen con mayor frecuencia.

\section{JULIANO Y LOS CULTOS PAGANOS TRADICIONALES}

Resultan abundantes y muy explícitos los pasajes de las Res gestae de Amiano en que se caracteriza la actitud religiosa del emperador Juliano, a lo que hay que añadir sus continuos esfuerzos por restaurar el culto tradicional ${ }^{20}$.

Como punto de partida hemos de tener en cuenta que dicho emperador se entregaría, de acuerdo con nuestro historiador, a la consulta y prácticas

\footnotetext{
${ }^{19}$ D. FlaCh (1972). "Von Tacitus zu Ammian". Historia, 21, p. 341.

${ }^{20}$ Para más detalles remitimos, entre otros, a R. L. RIKE. (1987). Apex omnium. Religio in the Res Gestae of Ammianus, Berkeley.
} 
correspondientes a los augures y arúspices (Amm.Marc. 21.2.4) ${ }^{21}$, así como a los restantes cultos que realizaban los adoradores de los dioses tradicionales.

Por ello no es de extrañar que Juliano, desde los primeros años de su juventud, sintiera una inclinación desmesurada hacia el culto de los dioses (Amm.Marc. 22.5.1).

Ya desde estos momentos iniciales la expresión amianea numinum cultus hemos de considerarla en conexión con el politeísmo tradicional, como lo demuestra claramente el edicto promulgado por el propio emperador a través del cual se prohibía al colectivo de los rétores cristianos ejercer sus enseñanzas si no adoptaban el culto a los dioses (Amm.Marc. 25.4.20).

En realidad estos numina son los mismos a los que se refiere Salustio, prefecto de las Galias, en la carta que dirige al emperador y en la que le conjura a suspender la expedición contra los persas y a no enfrentarse a un desastre seguro hasta que no haya implorado la benevolencia de los dioses (Amm.Marc. 23.5.4).

Algún tiempo después, con motivo de la retirada de las tropas romanas a través de Corduena en el año 363, con posterioridad a la orden emitida por el emperador para quemar la flota, el autor de Antioquía asegura: "como la sabiduría humana no ofrecía ninguna solución, habiendo permanecido durante mucho tiempo vacilantes e indecisos, levantaron altares e inmolaron víctimas para interrogar a los dioses acerca de sus designios" (Amm.Marc. 24.8.4) ${ }^{22}$.

\section{OTRAS MANIFESTACIONES DEL NUMEN}

De la prospectiva ofrecida por el escritor de Antioquía se deriva que las múltiples divinidades secundarias no constituyen más que simples parcelas del numen superior, del que proceden y reciben su poder a un mismo tiempo.

Como consecuencia de ello para nuestro historiador el numen se manifiesta bien a través de poderes superiores (superae potestates) (Amm.Marc. 25.3.17 y 28.4.24), bien mediante poderes sustanciales (substantiales potestates) (Amm.Marc. 21.1.8. Ver igualmente 14.11.25).

21 W. Liebeschuetz. (1988). "Ammianus, Julian and Divination", En Festschrift I.Opelt. Frankfurt 1988, p.198 y ss.

22 Al parecer el mismo Amiano, integrante de la comitiva imperial, se hallaría entre estos numinum cultores. 
En cualquier caso hay que contar con una omnipresencia de lo divino, que se hace manifiesta a través del desarrollo de los hechos históricos.

De esta manera los sucesos y acontecimientos de la vida cotidiana se hallarían regidos por la caelestis cura (Amm.Marc. 18.3.1), los decreta caelestia (Amm.Marc. 25.4.26), los caelestia adiumenta (Amm.Marc. 19.3.12), el nutus caelestis (Amm.Marc. 20.5.4 y 22.2.5), la caelestis ratio (Amm.Marc. 19.11.17) y el caeleste praesidium (Amm.Marc. 19.6.7).

Todos estos términos llevan implícito en sí la idea religiosa de integración y penetración permanente de lo divino en lo profano.

Junto a ello la supervivencia de los dioses tradicionales parece hallarse vinculada menos a la intensidad de los sentimientos religiosos que a las pretensiones de nuestro autor por enriquecer y adornar su estilo con las sutilezas y estética de la retórica (Ver, por ejemplo, Amm.Marc. 18.6.3; 21.5.1; 24.7.4; 28.1.1; 29.2.20-21; 31.1.1; 31.10.1; 31.13.1...).

Pero es que, además, los genios personales, así como los correspondientes al vulgo, se hallan integrados en la jerarquía extremadamente compleja de los poderes hipostáticos como emanación de la divinidad superior, de quien se concierten en presencia sensible en todo el universo ${ }^{23}$.

En este contexto posiblemente haya que incluir igualmente la importancia adquirida por el sol, así como la posible influencia que el historiador recibiría de la corriente filosófico-religiosa que veía en este astro el principio del mundo ${ }^{24}$.

De la misma manera hemos de hacer alusión aquí, aunque no desarrollemos el tema, a la importancia que el autor de Antioquía asigna a la astrología en el devenir de los acontecimientos de toda índole (Amm.Marc. 23.6.25: veridica vaticinandi (ides) $)^{25}$.

${ }^{23}$ G. ROCHEFORT. (1957). "La démonologie de Saloustios et ses rapports avec celle de l'empereur Julien". $B A G B, 4$, p. 53 y ss.

${ }^{24}$ P. M. CAMUS. (1967). Ammien Marcellin, témoin des courants culturels et religieux à la fin du IVe siècle, pp.139 y ss.

25 J. B. Clerc. (1989). "Á propos d'Ammien 23,6,5: une prétendue profession de foi en 1'astrologie". En Mélanges T. Zawadzk. Friburgo, pp.167 y ss. considera poco probable que dicha expresión haga alusión directa a la astrología, relacionándola más bien con la teurgia y los oráculos caldeos. 


\section{CONCLUSIONES}

A partir de estos testimonios de Amiano, ¿qué se puede deducir con respecto a sus creencias religiosas?

En primer lugar que, a pesar de que el emperador Juliano, figura principal de su obra histórica, al menos en lo que se refiere a los libros que de ella se nos conservan $^{26}$, se mostró como un defensor acérrimo de la religión tradicional, así como un preconizador feroz de la vuelta a la misma, es posible pensar que dicha descripción proceda de la creencia en un politeísmo jerarquizado, es decir de una situación intermedia entre el monoteísmo y el politeísmo.

A este respecto lo que parece que se encuentra en la base de las creencias del historiador antioqueno es una omnipresencia del elemento divino, siendo la divinidad la que se halla implícita en lo humano de forma constante, imbuyendo y penetrando por consiguiente todas las acciones de los humanos ${ }^{27}$.

Como manifestación de este principio, los sacrificios que se ofrecen a la divinidad implican en todos los casos una concepción activa de la misma.

Eso significa que, en el fondo, éstos no constituyen más que la forma suprema de petición a los dioses, así como de mantenerlos propicios y solícitos ${ }^{28}$.

Pero, junto a ello, no debemos olvidar que la teología de la historia en el autor de Antioquía no se fundamentaba exclusivamente en la inconstancia de la fortuna $^{29}$.

Es más, esta visión trágica del devenir histórico tendría su base en la contradicción existente entre la fe inquebrantable del hombre en la eternidad de

${ }^{26}$ Ver, entre otros, M. CALtABIANO. (1998). "Giuliano imperatore nelle Res Gestae di Ammiano Marcellino: tra panegirico e storia". Rudiae, 10, p. 335 y ss.

27 Más detalles, entre otros, en F. PASChOud (1988). "Justice et Providence chez Ammien Marcellin". En Miscellanea Calderone. Mesina, pp.139 y ss.

${ }^{28}$ P. M. CAMUS. Ammien Marcellin, témoin des courants culturels et religieux à la fin du IVe siècle, pp. 223 y ss.

${ }^{29}$ Ver, entre otros, T. D. BARNES. (1998). Ammianus Marcellinus and the Representation of Historical Reality. Ithaca, y N. SANTOS. "Fortuna y fatum: la contingencia en el desarrollo de la historia según Amiano Marcelino" (en prensa). 
Roma y la realidad de los hechos, que denotan una decadencia progresiva del Imperio sometido al asalto de los bárbaros ${ }^{30}$.

A lo que hace alusión constantemente Amiano es a una teología del poder, cuyo ejercicio resulta sacralizador y cuyo análisis lleva a cabo a través de sus manifestaciones maravillosas, que constituyen el fundamento de su orden social y al mismo tiempo la base de toda legitimidad política ${ }^{31}$.

Como consecuencia de ello lo maravilloso constituye el lenguaje más apropiado para tratar de explicar las relaciones de privilegio que unen al soberano con la divinidad mediante el ejercicio de un carisma de función ${ }^{32}$.

${ }^{30}$ I. LANA. (1998). "La vision tragique de l'histoire chez Ammien Marcellin". Pallas, 49, 237 y ss. Cf. V. ZARINI. (1999). "Histoire, panégyrique et poésie: trois éloges de Rome autour de l'an 400 (Ammien Marcellin, Claudien, Rutilius Namatianus)". Ktèma, 24, p. 167 y ss.

31 P. Siniscalco. (1989). "Le sacré et l'expérience de l'histoire. Ammien Marcellin et Paul Orose". $B A G B$, p. 355 y ss.

${ }^{32}$ M. MESLIN. "Le merveilleux comme langage politique chez Ammien Marcellin", p.363. 